\title{
Improving of fingerprint segmentation images based on K-means and DBSCAN clustering
}

\author{
El Mehdi Cherrat ${ }^{1}$, Rachid Alaoui ${ }^{2}$, Hassane Bouzahir ${ }^{3}$ \\ ${ }^{1,3}$ Laboratory of Systems Engineering and Information Technology, National School of Applied Sciences, \\ Ibn Zohr University in Agadir, Morocco \\ ${ }^{2}$ Laboratory of Systems Analysis, Information Processing and Integrated Management, \\ Higher School of Technology- Sale, Mohammed V University in Rabat, Morocco
}

\begin{tabular}{l}
\hline \hline Article Info \\
\hline Article history: \\
Received Aug 1, 2018 \\
Revised Dec 19, 2018 \\
Accepted Mar 4, 2019 \\
\hline
\end{tabular}

Keywords:

Classification

DBSCAN

Fingeprint segmentation image

K-means

Machine learning

\begin{abstract}
Nowadays, the fingerprint identification system is the most exploited sector of biometric. Fingerprint image segmentation is considered one of its first processing stage. Thus, this stage affects typically the feature extraction and matching process which leads to fingerprint recognition system with high accuracy. In this paper, three major steps are proposed. First, Soble and TopHat filtering method have been used to improve the quality of the fingerprint images. Then, for each local block in fingerprint image, an accurate separation of the foreground and background region is obtained by K-means clustering for combining 5-dimensional characteristics vector (variance, difference of mean, gradient coherence, ridge direction and energy spectrum). Additionally, in our approach, the local variance thresholding is used to reduce computing time for segmentation. Finally, we are combined to our system DBSCAN clustering which has been performed in order to overcome the drawbacks of $\mathrm{K}$-means classification in fingerprint images segmentation. The proposed algorithm is tested on four different databases. Experimental results demonstrate that our approach is significantly efficacy against some recently published techniques in terms of separation between the ridge and non-ridge region.
\end{abstract}

Copyright (C) 2019 Institute of Advanced Engineering and Science. All rights reserved.

\section{Corresponding Author:}

El mehdi cherrat,

Laboratory of Systems Engineering and Information Technology,

National School of Applied Sciences,

Ibn Zohr University in Agadir, Morocco.

Email: el.cherrat@gmail.com

\section{INTRODUCTION}

In present world, fingerprints have become an important biometric technology due to its uniqueness and invariance to every person. Moreover, with the popularity of fingerprinting technology, especially in mobile phones, the technology previously used in the field of criminal investigation is now commercialized. This biometric trait is more used and acceptable by the users because the capturing device is relatively small and identification accuracy is comparatively very high to other biometric recognition techniques such as the retina, iris, hand geometry, etc.[1-2].

The image segmentation is one of the main problems in the field of computer vision and image processing. Therefore, the fingerprint segmentation is typically the first and foremost step in the process of biometric recognition system based on fingerprint. In addition, the effect of this step directly affects the performance of the system.

The general structure of fingerprint recognition system consists of four major steps. In the first one, the acquisition of fingerprint image is process of getting a digitalised image of a person by using the specific sensors. These images can be acquired in two ways: offline and live-scan acquisition [3-4]. In the second 
step, the pre-processing is allowed to improve overall quality of the captured image. Through, it is frequently difficult to realize this process because the presence of large amount noisy areas in the image [5-6]. After that, the segmentation is applied. It is the process of separation in image into two regions: the region of the fingerprint image that contains all important data needed for recognition is called foreground region, while the regions which have been the blurred or noisy area are called background region. In the next step, the features points are extracted from a pre-processed fingerprint image such as ridge ending and bifurcation uniformly called minutiae. In the last step, generally, the matching of the extracted the feature points in order to perform the identification of the person.

Automatic segmentation has attracted considerable amount of reach interest in the last decade. Therefore, in this paper, improved fingerprint segmentation method using two machine learning models is presented. In our algorithm, we have been used particular filtering method to evaluate the quality of image acquired. After that, the fingerprint image is partitioned into non-overlapping blocks of particular size. Moreover, for each block, the feature vector is represented by its: variance, difference of mean, gradient coherence, ridge orientation and energy spectrum. Furthermore, the local variance thresholding is used to distinct between the features which will be computed or considered as null. The first machine learning, $\mathrm{K}$-means classifier, is trained for dividing each extracted feature into two classes (foreground area and background area). Finally, the second one (DBSCAN clustering) is used to remove some misclassified blocks due to K-means classification. Thus, the contour smoothing is performed to enhance the images segmented of fingerprints. The rest of the paper is separated into four sections. In the section 2, the related works in the field are reviewed. Section 3 discusses the proposed segmentation algorithm. Experimental results for four databases have been analysed and discussed in Section 4. Finally, the conclusion is presented in the last section.

\section{RELATED WORKS}

The fingerprint image segmentation is one of the principal stage for automated fingerprint recognition system. This pre-processing stage allows to separate the fingerprint region from a captured image with two areas: foreground and background [7]. Most existing techniques of segmentation are based on the feature of pixel intensity in a block because it is computationally faster than others based only on the pixel intensity [8-10]. For fingerprint segmentation, there are so various methods have been proposed in the stateof-the-art. Here, we briefly review these methods.

$\mathrm{Li}$, et al. [11] proposed a segmentation technique by calculating gray contract and Fourier spectrum energy ratio for each block in fingerprint image and then classified these block by linear support vector machine approach. Finally, morphological operations are used to improve the segmented image. Akram, et al. presented a segmentation method by computing mean, variance and gradient deviation information of each block in fingerprint image. The segmentation image of fingerprint is obtained by using the linear classifier [12]. Li, et al. [13] suggested a method for fingerprint image segmentation using a novel approach of K-Means. the fingerprint image is divided into non-overlapping blocks. Furthermore, for each block, the variance, direction and energy spectrum are extracted to construct feature vectors and then, classified these characteristics by K-means clustering algorithm. Finally, used the post-processing to remove the remaining isolated blocks in foreground or background region. In Yang, et al. [14-15] have been subjected a novel algorithm of fingerprint images segmentation by using an unsupervised learning method based on K-means classifier. Thus, for each block, the average and coherence data is computed in order to divide the image into two regions by using K-means clustering approach. The correlation based fingerprint image segmentation is used in [16]. Fahmy, et al. [17] proposed a technique that utilizes morphological processing to extract the foreground from the fingerprint image. After the division of image into nonoverlapping blocks, this method used the feature vector for each block to realize fingerprint segmentation. Then, the adaptive thresholding is used to convert the fingerprint image to a binary one. Next, some morphological operations (closing and opening) are applied, to segmented the image. Finally, the complex Fourier series expansion are performed to smooth the segmented contour. In this algorithm, the image is separated into blocks and sub-blocks. Afterwards, the thresholding level have been applied for segmentation. Das, et al. [18] achieved the fingerprint segmentation by computing block based statistics and morphological operations. Abboud, et al. [19] presented a new segmentation technique by statistical computing: mean, variance and coherence features of each block in fingerprint image based on an automatic threshold values and Otsu's method. Finally, the filing the gaps is applied to remove the noise in some regions in foreground or background by using particular sets of rules based on neighboring regions. 


\section{PROPOSED APPROACH}

Our proposed method is improved the fingerprint image segmentation based on K-Means and DBSCAN clustering. A robust and effective fingerprint image segmentation algorithm is important phase for a fingerprint recognition system. In this section, we detail the proposed technique which is illustrated in Figure 1 . The details of each phase are represented in the following.

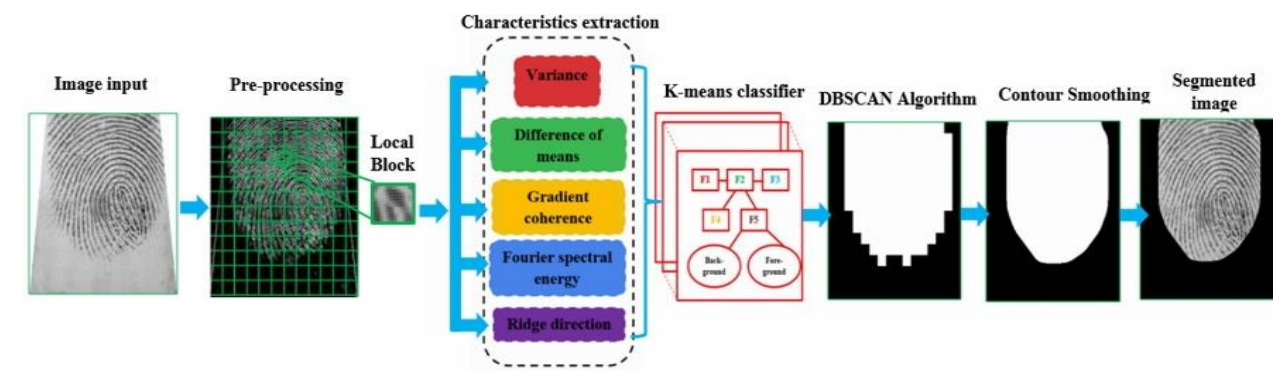

Figure 1. Block diagram of proposed algorithm for fingerprint image segmentation

\subsection{Pre-processing}

In this phase, Sobel and TopHat filter method have been used to improve the quality of the fingerprint image. Sobel structuring operators Sobel $x$ and Sobel $y$ for image are represented in (1).

$$
\text { Sobel }_{x}=\left(\begin{array}{ccc}
-1 & 0 & 1 \\
-2 & 0 & 2 \\
-1 & 0 & 1
\end{array}\right), \text { Sobel }_{y}=\left(\begin{array}{ccc}
-1 & -2 & -1 \\
0 & 0 & 0 \\
1 & 2 & 1
\end{array}\right)
$$

The gradient $G_{x}$ and gradient $G_{y}$ of pixels are defined from image $I_{m g}$ by (2).

$$
G_{x}=\text { Sobel }_{\mathrm{x}} * \mathrm{I}_{\mathrm{mg}}, \quad G_{y}=\text { Sobel }_{\mathrm{y}} * \mathrm{I}_{\mathrm{mg}}
$$

The result of gradient is combined to find the absolute magnitude (the output edge). This result is described as follows:

$$
G(x, y)=\sqrt{\mathrm{G}_{\mathrm{x}}^{2}+\mathrm{G}_{\mathrm{y}}^{2}}
$$

The fingerprint image is ameliorated after normalisation and Sobel technique. However, the fingerprint image is more improved by using the TopHat technique. This filter is a process that extracts details and small elements from image. TopHat filtering is based on dilation, erosion, opening and closing method. The morphological dilation and erosion operation for image $\mathrm{I}_{\mathrm{mg}}$ of size $x \times y$ with structuring element Se are defined by (4) and (5) respectively:

$$
\begin{aligned}
& {\left[I_{m g} \oplus S_{e}\right](x, y)=\max _{(\mathrm{s}, \mathrm{t}) \in \mathrm{S}_{\mathrm{e}}}\left\{I_{m g}(x+s, y+t)\right\}} \\
& {\left[I_{m g} \ominus S_{e}\right](x, y)=\min _{(\mathrm{s}, \mathrm{t}) \in \mathrm{S}_{\mathrm{e}}}\left\{I_{m g}(x+s, y+t)\right\}}
\end{aligned}
$$

The opening and closing process for image $I_{m g}$ with structuring element $S_{e}$ are described by combining the erosion and dilatation operation given by (6) and (7) respectively:

$$
\begin{aligned}
& I_{m g} \circ S_{e}=\left(I_{m g} \ominus S_{e}\right) \oplus S_{e} \\
& I_{m g} \bullet S_{e}=\left(I_{m g} \oplus S_{e}\right) \ominus S_{e}
\end{aligned}
$$

The opening $\mathrm{TopHa}_{\text {top }}$ and closing $\mathrm{TopHat}_{c l}$ operations for image Img with structuring element Se are represented by (8) and (9) respectively:

$$
\begin{aligned}
& \operatorname{TopHat}_{o p}\left(I_{m g}\right)=I_{m g}-\left(I_{m g} \circ S_{e}\right) \\
& \operatorname{TopHat}_{c l}\left(I_{m g}\right)=I_{m g}-\left(I_{m g} \bullet S_{e}\right)
\end{aligned}
$$

Improving of fingerprint segmentation images based on K-means and DBSCAN... (El mehdi Cherrat) 


\subsection{Segmentation}

After the pre-processing phase, the fingerprint image is divided into non-overlapping local blocks of size $w \times w$. Further, to every block, the characteristic vector is classified into two classes: foreground and background region by using K-means classification.

\subsubsection{Characteristics extraction}

The characteristic vector is represented, for each block in fingerprint image, by its three categories namely: image intensity based characteristics, gradient based characteristics and ridge based characteristics.

\section{a. Image intensity based characteristics}

The change in intensity values is usually specific along the ridges and no-ridges when compared to background areas in fingerprint image. General image intensity based characteristics can be used to define the most intensity such as difference of mean, which is the difference between the local intensity mean and the global intensity mean, and variance blocks in given image $I_{m g}$ of size $x \times y$. These proprieties are computed by (11) and (12) respectively.

$$
\begin{aligned}
& I_{\text {mgMeanL }}(x, y)=\frac{1}{\mathrm{~W}^{2}} \sum_{x=1}^{\mathrm{w}} \sum_{y=1}^{\mathrm{w}} \mathrm{I}_{\mathrm{mg}}(x, y) \\
& \operatorname{Ch}_{\text {DiffMean }}(x, y)=I_{\text {mgMeanL }}(x, y)-I_{\text {mgMeanG }}
\end{aligned}
$$

where $I_{m g M e a n L}$ is the mean intensity of the local block of image and $I_{m g M e a n G}$ is the mean intensity of the global image.

$$
C h_{V a r}(x, y)=\frac{1}{W^{2}} \sum_{x=1}^{w} \sum_{y=1}^{w}\left(I_{m g}(x, y)-I_{\text {mgMeanL }}\right)^{2}
$$

\section{b. Gradient based characteristics}

The gradient is utilized to obtain the directional variation in intensity value along a direction of image $I_{m g}$ of size $x \times y$. Characteristics such as gradient cohrence and ridge direction can be classified into gradient based characteristics. The gradient coherence and ridge direction features are calculated by (16) and (20) respectively.

$$
C h_{\text {Coherence }}(x, y)=\sqrt{\frac{\left(G_{\text {Cohx }}(x, y)-G_{\text {Cohy }}(x, y)\right)+4 G_{\text {Cohxy }}(x, y)^{2}}{G_{\text {Cohx }}(x, y)+G_{\text {Cohy }}(x, y)}}
$$

Where

$$
\begin{aligned}
& G_{\text {Cohx }}(x, y)=\sum_{x=1}^{w} \sum_{y=1}^{w}\left(G_{x}^{2}(x, y)\right) \\
& G_{\text {Cohy }}(x, y)=\sum_{x=1}^{w} \sum_{y=1}^{w}\left(G_{y}^{2}(x, y)\right) \\
& G_{\text {Cohxy }}(x, y)=\sum_{x=1}^{w} \sum_{y=1}^{w}\left(G_{x}^{2}(x, y) * G_{y}^{2}(x, y)\right)
\end{aligned}
$$

The gradient $G_{x}$ and gradient $G_{y}$ are defined by (3).

$$
\begin{aligned}
& S_{q 1}=\sum_{x=1}^{w} \sum_{y=1}^{w}\left(G_{x}^{2}(x, y)-G_{y}^{2}(x, y)\right) \\
& S_{q 2}=\sum_{x=1}^{w} \sum_{y=1}^{w} 2 \mathrm{G}_{\mathrm{x}}(x, y)^{*} \mathrm{G}_{\mathrm{y}}(x, y) \\
& D(x, y)=\frac{1}{2} \tan ^{-1}\left(\frac{S q_{2}}{S q_{1}}\right)
\end{aligned}
$$




$$
C h_{\text {Direct }}(x, y)= \begin{cases}\frac{\pi}{4} & \mathrm{Sq}_{1}=0, \mathrm{Sq}_{2}<0 \\ \frac{3 \pi}{4} & \mathrm{Sq}_{1}=0, \mathrm{Sq}_{2} \geq 0 \\ \mathrm{D}(\mathrm{x}, \mathrm{y})+\frac{\pi}{2} & \mathrm{Sq}_{1}>0 \\ \mathrm{D}(\mathrm{x}, \mathrm{y}) & \mathrm{Sq}_{1}<0, \mathrm{Sq}_{2} \leq 0 \\ \mathrm{D}(\mathrm{x}, \mathrm{y})+\pi & \mathrm{Sq}_{1}<0, \mathrm{Sq}_{2}>0\end{cases}
$$

\section{c. Gradient based characteristics}

Characteristics such as ridge frequency (energy spectrum) which is computes by using Fourier transform for each local block of image $\mathrm{I}_{\mathrm{mg}}$ of size $x \times y$ can be grouped as ridge based characteristics. This feature is compute by (22).

$$
\begin{aligned}
& F(k, l)=\sum_{x=1}^{w} \sum_{y=1}^{w} I_{m g}(x, y) e^{-i 2 \pi\left(\frac{k x}{w}+\frac{l y}{w}\right)} \\
& C h_{\text {Freq }}(x, y)=\sqrt{R E(F(k, l))^{2}+\operatorname{IM}(F(k, l))^{2}}
\end{aligned}
$$

where $\mathrm{k}, \mathrm{l} \in\{1, \ldots . . w\}$ and $<(\mathrm{x}, \mathrm{y})(\mathrm{k}, \mathrm{l})>=\mathrm{xk}+\mathrm{yl}$.

\subsubsection{Local variance thresholding}

The variance local thresholding decides whether or not the region in fingerprint image needs to compute another characteristic for segmentation. The local variance is calculated in given image $I_{m g}$ of size $x \times y$ by (12). If local intensity variance in a $w \times w$ image block is greater than 0 , the other characteristic of the block will be computed otherwise are considered as null. Therefore, from Table 1, in DB1 it is observed that the difference of the processing time of our proposed segmentation is achieved almost 10 seconds for an obtained fingerprint image. Additionally, it is shown that the average segmentation time in 4 databases is less than other method based on K-means segmentation which the block is represented just by 3-dimensional characteristic vector [13].

Table 1. Comparison of segmentation time in second for each database in FVC2004

\begin{tabular}{ccc}
\hline Databases & K-means with 3 features [13] & Our Algorithm with 5 features \\
\hline DB1 & 17,46 & $\mathbf{7 , 9 5}$ \\
DB2 & $\mathbf{1 7 , 5 2}$ & 18,66 \\
DB3 & $\mathbf{1 7 , 7 8}$ & 18,30 \\
DB4 & $\mathbf{1 7 , 6 8}$ & 19,66 \\
Avg & 17,61 & $\mathbf{1 6 , 1 4}$ \\
\hline
\end{tabular}

\subsubsection{K-means clustering}

In our proposed method, K-means classifier is utilized to classify the five extracted characteristics, variance, difference of mean, gradient coherence, ridge direction and energy spectrum, from each local block in fingerprint image in order to distinct the foreground area to the noisy background area. The characteristic extraction vector for each block is represented by (23). On the one hand, the K-means algorithm is a popularly unsupervised machine learning models [20] used for clustering technique of the data [21]. On the other hand, it is simplified to implementation, eased of interpretation, faster and adapted to sparse data. $\mathrm{K}$-means classification is represented as follows. Firstly, this approach selects randomly the number of clusters and assigns the cluster with closest centroids; then it determines each data point to the nearest centroids and for every cluster, the new centroids are recalculated, and this process is repeated until some condition is verified [22].

$$
\mathrm{Ch}_{\text {Vector }}(x, y)=\left[C h_{\text {Diffmean }}, C h_{\text {Var }}, C h_{\text {Coherence }}, C h_{\text {Direct }}, C h_{\text {Freq }}\right]
$$

\subsubsection{DBSCAN algorithm}

DBSCAN, Density-Based Spatial Clustering of Applications with Noise, is another model of machine learning and clustering analysis algorithm proposed by Martin Ester et al. [23]. DBSCAN is one of the most commonly used cluster analysis algorithms. This algorithm is density-based: given a set of points in a dataset, the algorithm can the nearby points are grouped together (points with many adjacent points) and the outliers in the low density area are marked. The general algorithm of DBSCAN Clustering is shown in 
algorithm 2. The reason of using DBSCAN over other clustering algorithms is that it does not demand a fixed number of groups in dataset. It also recognizes outliers as noise which simply introduces them into the cluster even if the data points are very different. In addition, it is a good place to find groups of any size and shape. In our propsed algorithm, DBSCAN Clustering is used to achieve more compact blocks for reducing the misclassification region due to the K-means algorithm .Figure 2 presents the result using the DBSCAN algorithm. Finaly, the contour smoothing (filtering in a complex Fourier transform domain [24]) as postprocessing technique to smoothen the edges of mask [17].
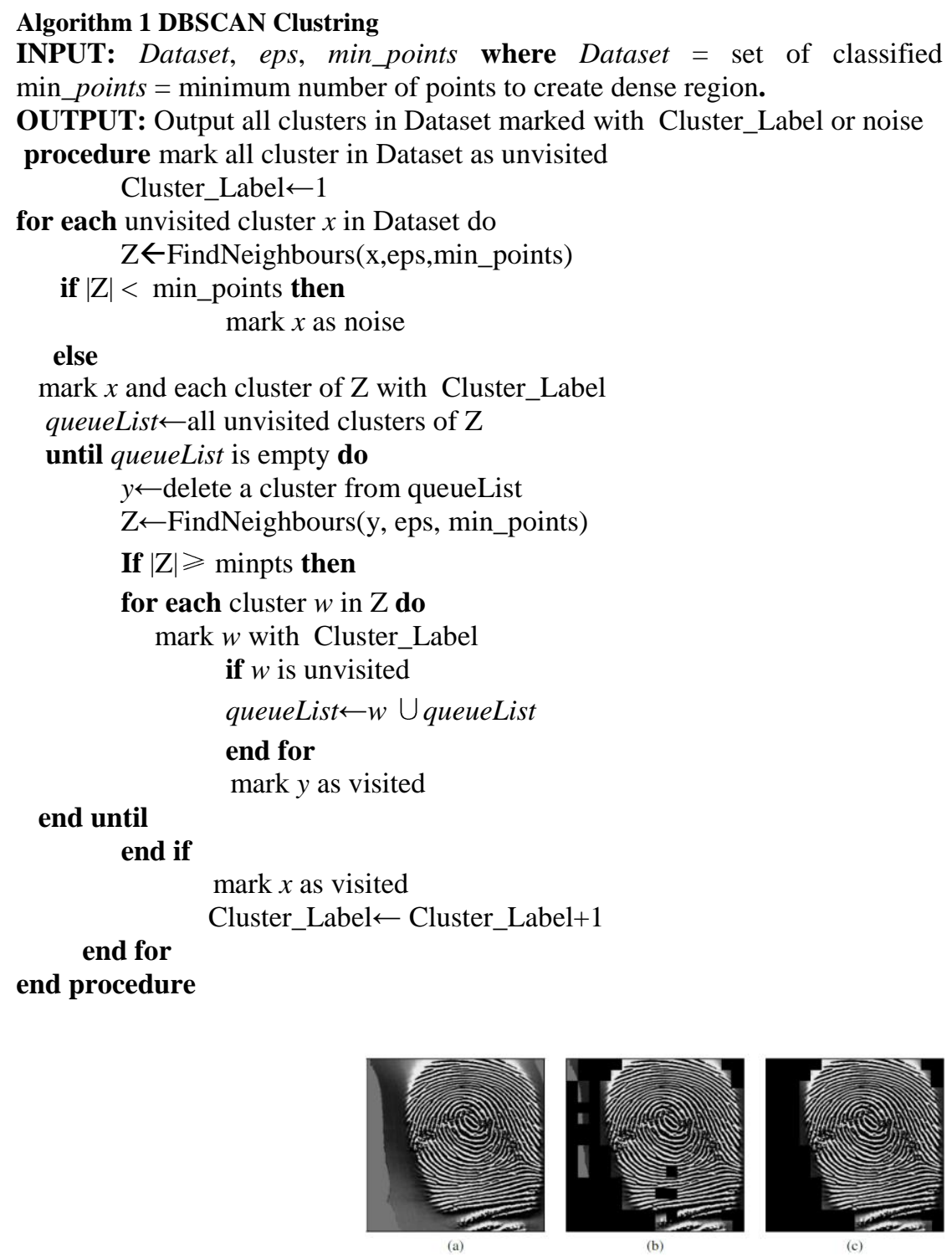

Figure 2. Removing the misclassification region by DBSCAN algorithm:

(a) Original image; (b) Our K-means classification; (c) Our K-means and DBSCAN classification

\section{RESULTS AND DISCUSSION}

The experimental operation platform in this study is described as follows: the host configuration: CPU Intel Core2 Duo at 2.00 GHz, RAM 3.00 GB, runtime environment: Microsoft Visual Studio C++ 2013 with OpenCV library. In order to better verify our algorithm, the following segmentation methods are adopted in the experiment: SVM [9], K-means with 3-dimension feature [13], MP [17], ACT [19]. These segmentation algorithms were compared to each other. In order tovalidate the proposed algorithm, the results have been tested on the public Fingerprint Verification Competition 2004 dataset [25] which contains 4 databases, namely DB1, DB2, DB3 and DB4. The performance measure is used the number of misclassification as defined by (25). 


$$
\begin{aligned}
& \operatorname{Prob}_{1}=\frac{N b r_{b e}}{N b r_{b}}, \operatorname{Prob}_{2}=\frac{N b r_{f e}}{N b r_{f}} \\
& \operatorname{Prob}_{E r r}=\operatorname{Avg}\left(\operatorname{Prob}_{1}, \operatorname{Prob}_{12}\right)
\end{aligned}
$$

where $N b r_{b e}$ is number of background classified error, $N b_{r b}$ is the total number of true background regions in the fingerprint image and $\mathrm{Prob}_{1}$ is the probability that a foreground region is classified as background. $\mathrm{Nbr} f \mathrm{fe}$ is the number of foreground classified error, $N b r_{f}$ is the total number of true foreground regions in the fingerprint image and $\mathrm{Prob}_{2}$ is the probability that a background region is classified as foreground. The probability of error $\mathrm{Prob}_{\mathrm{Err}}$ is the average of $\mathrm{Prob}_{1}$ and $\mathrm{Prob}_{2}$.

The existing algorithms of fingerprint image segmentation, SVM [11], K-Means with 3-dimension feature [13], MP [17] and ATC [19], are implemented for comparison of segmentation performance. The comparison be tween proposed method and others works in measure of average segmentation error for fingerprint images at different databases is shown in Table 2. From Table 2, in The SVM [11] and K-Means with 3-dimension feature [13], the corresponding value for misclassification rate in DB1 is $18,75 \%, 20,28 \%$ respectively. Furthermore, the error rate of segmentation in MP [17], ATC [19] and our proposed method is $0,28 \%, 13,31 \%$ and $0,30 \%$ respectively. In the second database, if SVM [11], K-Means with 3-dimension feature [13], MP[17] and ATC [19] give 34,56\%, 22,30\%, 2,92\%, 29,79\% respectively in segmentation rate however our proposed method takes minimum error rate as $1,66 \%$. Likewise, in the last database, misclassification rate $(1,09 \%)$ is clearly less for the proposed algorithm compared to other techniques in the third database. SVM [11] has failed to classify better the forwound and background region (error rate as $28,89 \%$ ) in the fourth database. thus, our system has succeeded to reduce $0,63 \%$ of segmentation error than K-Means with 3-dimensions features [13], MP [17] and ATC [19] which give 5,06\%, 1,31\% and 17,36\% respectively. The proposed algorithm results show a better performance respect to other algorithms with average misclassification rate as $0,67 \%$ at different databases of fingerprint images. The means of these results shows that our algorithm is improved the recognition accurate rate of the person.From these existing works, it is worthy to say that the results of proposed method are clearly superior in term segmentation error rate. The Figure 3 represents the visual results of the proposed algorithm and the other techniques. When we compared to the existing works in this figure. We can say that our proposed is efficient and reduced error rate of segmentation.The visual quality results of segmented image indicate that the proposed algorithm adapts and gives better results in term of segmentation in different environments than the comparative techniques for accuracy segmentation.

Table 2. Comparison of segmentation misclassification using different algorithms in FVC2004

\begin{tabular}{cccccc}
\hline Databases & SVM [11] & K-means [13] & MP [17] & ATC [19] & Proposed Algorithm \\
\hline D1 & $18,75 \%$ & $20,28 \%$ & $\mathbf{0 , 2 8 \%}$ & $13,31 \%$ & $0,30 \%$ \\
D2 & $34,56 \%$ & $22,30 \%$ & $2,92 \%$ & $29,79 \%$ & $\mathbf{1 , 6 6 \%}$ \\
D3 & $1,32 \%$ & $12,60 \%$ & $2,54 \%$ & $19.44 \%$ & $\mathbf{1 , 0 9 \%}$ \\
D4 & $28,89 \%$ & $5,06 \%$ & $1,31 \%$ & $17,36 \%$ & $\mathbf{0 , 6 3 \%}$ \\
Avg & $28,38 \%$ & $15,06 \%$ & $1,76 \%$ & $19,98 \%$ & $\mathbf{0 , 9 2 \%}$
\end{tabular}

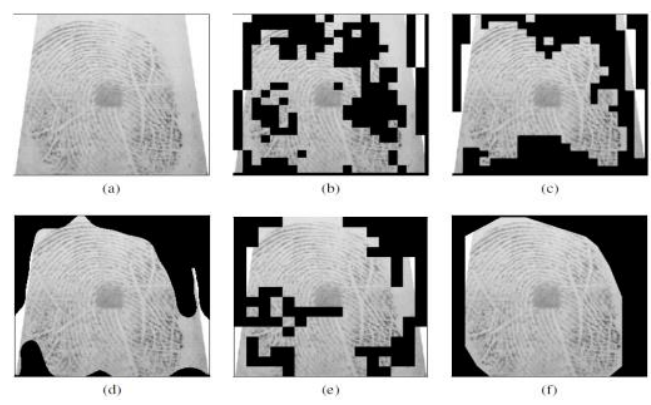

Figure 3. Segmentation results using different methods in DB2:

(a) Original image, (b) SVM [11], (c) K-means [13], (d) MP [17], (e) ATC [19], (f) Proposed method

\section{CONCLUSION}

This paper has proposed an improved method of fingerprint segmentation images based on K-Means classification and DBSCAN clustering. Our proposed system is presented in three steps. In the first step, the quality of fingerprint images is enhanced using Soble and TopHat filtering method. In the second step,

Improving of fingerprint segmentation images based on K-means and DBSCAN... (El mehdi Cherrat) 
K-means approach is applied for each local block to classify the image into foreground and background region using five-dimensional characteristic vector extraction. Moreover, the processing time of segmentation is faster than another algorithm based on just 3 dimensions features due to the local variance thresholding. In the final step, DBSCAN algorithm is used to remove some misclassified blocks due to the $\mathrm{K}$-means clustering and contour smoothing is achieved to improve the image segmented. Simulation results show significantly that the proposed method is efficacy some recent existing techniques in average segmentation error rate. Therefore, it affects the performance of the system to have a higher accurate recognition rate of the person.

\section{REFERENCES}

[1] E.M Cherrat, R. Alaoui, H. Bouzahir, W. Jenkal, "High density salt-and-pepper noise suppression using adaptive dual threshold decision based algorithm in fingerprint images," IEEE Intelligent Systems \&Computer Vision, pp. 1-4, 2017.

[2] S.R. Borra, G.J Reddy, E. S. Reddy, "An Efficient Fingerprint Identification using Neural Network and BAT Algorithm," International Journal of Electrical and Computer Engineering (IJECE), vol. 8, no. 2, pp. 1194-1213, 2018.

[3] P. Wang,"Pattern recognition, machine intelligence and biometrics," Springer Berlin Heidelberg, 2011.

[4] A. Ross and J. Anil, "Biometric sensor interoperability: A case study in fingerprints," In ECCV Workshop BioAW, pp. 134-145, 2004.

[5] M. N. Abdulwahed and A. kamil Ahmed, "Underwater Image de-nosing using discrete wavelet transform and prewhitening filter," TELKOMNIKA Telecommunication Computing Electronics and Control, vol. 16, no. 6, 2018.

[6] R.M. Zayed, "Optimum Image Filters for Various Types of Noise," TELKOMNIKA Telecommunication Computing Electronics and Control, vol. 16, no. 5, 2018.

[7] B. Stojanovi, O. Marques, and A. Nekovi, "Latent overlapped fingerprint separation: a review," Multimedia Tools and Applications, 76(15), pp.16263-16290, 2017.

[8] C. Wu, S. Tulyakov, V. Govindaraju, "Robust point-based feature fingerprint segmentation algorithm," Advances in Biometrics, pp.1095-1103, 2007.

[9] E. Zhu, J. Yin, C. Hu, G. Zhang, "A systematic method for fingerprint ridge orientation estimation and image segmentation,". Pattern Recognition, 39(8), pp.1452-1472, 2006.

[10] N.V. Boulgouris, K.N. Plataniotis, E. Micheli-Tzanakou, "Biometrics: theory, methods, and applications," John Wiley \& Sons, (Vol. 9), 2009.

[11] Y. X. Li, X.Q. Peng and S. Li, "Fingerprint image segmentation method based on linear support vector machine," Application Research of Computers, 25(12), pp. 3669-3670, 2008.

[12] M. U. Akram, A. Ayaz and J. Imtiaz, "Morphological and gradient based fingerprint image segmentation", International Conference on Information and Communication Technologies (ICICT), pp. 14, 2011.

[13] H. Li and Y. Ping, "A novel approach of K-means based fingerprint segmentation algorithm", In Instrumentation \& Measurement, Sensor Network and Automation, IEEE International Symposium on, Vol. 1, pp. 218-221, 2012.

[14] G. Yang, G.-T. Zhou, Y. Yin, and X. Yang, "K-Means Based Fingerprint Segmentation with Sensor Interoperability," EURASIP J. Adv.Signal Process, vol. 2010, no. 1, p. 729378, 2010.

[15] G. Yang, Y. Li, Y. Yin, and Y.-S. Li, "Two-Level Evaluation on Sensor Interoperability of Features in Fingerprint Image Segmentation," Sensors, vol. 12, no. 12, pp. 31863199, 2012.

[16] S. R.Suralkar and P. M. Patil, "Correlation based Fingerprint Image Segmentation," Int. J. Comput. Appl., vol. 68, no. 7, pp. 13, 2013.

[17] M. F. Fahmy and M. A. Thabet, "A fingerprint segmentation technique based on morphological processing," In Signal Processing and Information Technology (ISSPIT), IEEE International Symposium on, pp. 000215-000220, 2013.

[18] D. Das and S. Mukhopadhyay, "Fingerprint Image Segmentation Using Block-Based Statistics and Morphological Filtering," Arab. J. Sci. Eng., vol. 40, no. 11, pp. 31613171, 2015.

[19] A.A, Abbood,S. Ghazali, A.R, Atheer Akram and U.P. Sabine, "Segmentation and enhancement of fingerprint images based on automatic threshold calculations," In International Conference of Reliable Information and Communication Technology Springer, pp.400-411, 2017.

[20] V. Mhaske-Dhamdhere and S. Vanjale, "A novel approach for phishing emails real time classification using k-means algorithm," International Journal of Engineering and Technology, 7, no. 1.2 , 96-100, 2018.

[21] G. Gan and M.K.P. Ng, "k-means clustering with outlier removal," Pattern Recognition Letters, 90, pp.8-14, 2017.

[22] J. Qin, W. Fu, H. Gao and W.X. Zheng, "Distributed K-Means Algorithm and Fuzzy C-Means Algorithm for Sensor Networks Based on Multiagent Consensus Theory," IEEE transactions on cybernetics, 47(3), pp.772-783, 2017.

[23] Ester, Martin, H.P Kriegel, J. Sander, X. Xu, "A density-based algorithm for discovering clusters in large spatial databases with noise," In Kdd, vol. 96, no. 34, pp. 226-231, 1996.

[24] R. C. Gonzalez and R. E. Woods, "Digial Image Processing," Prentice Hall, 2003.

[25] D. Maio, D. Maltoni, R. Cappelli et al, "Fvc2004: Third fingerprint verification competition biometric authentication," Springer Berlin Heidelberg, pp. 1-7, 2004. 